\title{
Educación intercultural en el contexto Latinoamericano. Una mirada a la Etnomatemática.
}

\author{
Intercultural education in the Latin America \\ context. A look to the Ethnomathematics. \\ Elia Calderón Leyton \\ Facultad de Ciencias Sociales y Humanas. \\ Universidad de Medellín. \\ eliacalderon.le@gmail.com
}

\begin{abstract}
Resumen
Este artículo se basa en el análisis de la interculturalidad y la Pedagogía intercultural etnomátemática que se han creado en Latinoamérica para dar respuesta a la demanda en educación intercultural, haciendo referencia a los pueblos indígenas y afrodescendientes en Latinoamérica. El objetivo de este artículo es presentar la Etnomatemática como curso en el programa de formación de profesores indígenas de la Licenciatura en Pedagogía de la Madre Tierra (LPMT) de la Universidad de Antioquia, Colombia. El problema que se discute es la confrontación de saberes y conocimientos de personas pertenecientes a esferas culturales diferentes, en el marco de la educación intercultural en el contexto latinoamericano. Por último, se plantean posibles soluciones para mejorar la educación intercultural y lograr su revitalización.
\end{abstract}

Palabras clave: Interculturalidad, Educación, indígenas, afrodescendientes, Latinoamérica

\begin{abstract}
This article is based on the analysis of the interculturality and the intercultural entomatical pedagogy created to respond to demands on Intercultural Education by referring to the indigenous and Afrodescendant peoples in Latin America. The objective of this article is to present the Ethnomathematics as a course in the training program for indigenous teachers of the Bachelor of Pedagogy of Mother Earth (BPME) of the University of Antioquia, Colombia. The discussed problem is the knowledge confrontation of persons who belong to different cultural domains, in the framework of intercultural education in the Latin America context. Finally, certain possible solutions to improve the Intercultural education and to the revitalization.
\end{abstract}

Keywords: Interculturality, education, indigenous, Afrodescendant, Latin America

Recibido: 28 de Junio de 2019 • Aceptado: 05 de Agosto de 2019

Elia Calderón Leyton

Educación intercultural en el contexto Latinoamericano. Una mirada a la Etnomatemática. Autoctonía. Revista de Ciencias Sociales e Historia, Vol. III, N² 2, Julio-Diciembre 2019, 244-267 ISSN 0719-8213

DOI: http://doi.org/10.23854/autoc.v3i2.119 


\section{Introducción}

Este trabajo se centra dentro de un contexto investigativo realizado en el marco que hemos venido desarrollando con varios profesores desde 2016 y que forma parte del Grupo de investigación en conflicto y paz, línea paz y subjetividad, de la Universidad de Medellín, sobre las pedagogías interculturales y educación en el continente. Por ello, hemos realizado una amplia revisión bibliográfica sobre la educación intercultural en América Latina, especialmente en Colombia en las últimas décadas. Además hemos efectuado entrevistas con especialistas y militantes de movimientos sociales de diferentes procedencias con el fin de analizar la incorporación de la interculturalidad en la educación.

Paralelamente también hemos organizado congresos, seminarios y encuentros sobre dicha temática. El análisis de los datos recolectados permitió no sólo identificar las tensiones que traspasaban la discusión, sino también las propuestas y soluciones surgidas sobre educación intercultural etnomatemática en la Universidad de Antioquia. Es por ello que pensamos que es relevante analizar la pedagogía intercultural como solución para revitalizar la educación intercultural en nuestro continente latinoamericano. Por lo tanto, es necesario enfocarnos luego en la pedagogía intercultural etnomatemática de la Universidad de Antioquia como un posible camino para revitalizar a la educación intercultural en América Latina.
Cabe recordar que las políticas educativas que se desempeñan en materias de cuestiones de interculturalidad, por lo general plantean un esfuerzo por diferenciar la Educación Intercultural Bilingüe (EIB), o en su defecto la etnoeducación, como una modalidad educativa de los sistemas nacionales para atender a los miembros de pueblos indígenas y poblaciones afro descendientes, de la noción de interculturalidad como un enfoque transversal de las políticas educativas. Recordemos que ambos son maneras de albergar las diversidades culturales existentes en los países en materia de educación. Generalmente se parte como marco referencial a la interculturalidad como enfoque en el abordaje de las políticas públicas (CEPAL 2017; Corbetta, 2016; López, 2009, 2006) con un matiz en los aportes de la interculturalidad crítica (Tubino, 2005; Walsh, 2009).

Intrínsecamente se parte de una visión que presenta la relevancia de interpelar a los Estados y sus actuales políticas a la hora de hacer efectivo el derecho a la educación de los mencionados pueblos, además de desarticular los históricos procesos de exclusión de los cuales siguen siendo a veces víctimas. Recordemos que la educación ha sido considerada por los organismos de Expertos sobre los Derechos de los Pueblos Indígenas como un derecho humano y un instrumento indispensable para lograr el goce de otros derechos humanos y las libertades fundamentales. Paralelamente vendría a ser una forma fundamental para que los pueblos socioeconómicamente marginados puedan salir de la pobreza y 
acceder a los medios que les permitan participar plenamente en sus comunidades.

Pero es imprescindible el rol que los Estados poseen como garantes para asegurar también otros derechos que cumplan con su obligación de hacer una educación de calidad para todas las personas pertenecientes a pueblos indígenas $\mathrm{y}$ poblaciones afrodescendientes en cada región determinada. Por otro lado, es necesario destacar las dos caras de la moneda que busca empoderar a la educación en la propia clave cultural que tanto pueblos indígenas como poblaciones afrodescendientes reclaman para sí. En otros términos, la "educación para sí" alberga por lo menos dos corrientes: la Educación Intercultural Bilingüe (EIB) y/o la etnoeducación que demanda, a pesar de sus diferentes tendencias en su base, educar a sus miembros en sus propias lenguas y cultura. Este reclamo exige que ambos pueblos tengan el derecho a que los Estados les garanticen una política educativa que asegure la calidad de la educación por medio de la ejecución de estos derechos.

Por ello, es fundamental evaluar esta educación de manera comparativa respecto al desarrollo de la EIB en la región y los desafíos principales que enfrentan los países latinoamericanos en la actualidad. Se trata de corrientes actuales en relación a la Educación Intercultural Bilingüe y a la interculturalidad en educación. Dentro de estas temáticas generalmente se enfrentan perspectivas de desarrollo hegemónico y otras formas de concebir la relación entre ecosistemas y cultura (Maya, 2013), por lo cual entran en conflicto (OREALC/UNESCO, 2017).

A pesar de que cabe resaltar que dentro de estas perspectivas se cristalizan tendencias en función de las políticas en el campo educativo y de las experiencias de países latinoamericanos, de las cuales cabe distinguir diferencias político-ideológicas. Dentro de estas corrientes existen diferentes modelos de sociedad con sus especiales contextos históricos que sustentan a las relaciones entre grupos sociocultural y lingüísticamente. Cabe recordar que dentro de América Latina, estas discusiones tienen como antecedente demandas históricas, desarrollos y experiencias pedagógicas previas que contextualizan la emergencia de la EIB. Por ejemplo, el modelo hegemónico, basado epistemológicamente desde el saber científico occidental, tiende a construir una forma de conocimiento y parte de verdades que deslegitiman toda otra forma de producir conocimiento.

Es por ello que los debates sobre la educación se rigen desde la diversidad en América Latina a partir de estos diversos modelos. Estas corrientes parten desde maneras de inclusión/ integración a la cultura nacional y estrategias de interculturalizar el sistema educativo en general, donde se considera la necesidad de incluir “alternativas epistémicas" en las aulas, donde estos saberes son considerados alternativos al conocimiento occidental, pero como formas alternativas de producir saberes que sustentan las prácticas sociales, culturales y educativas de las 
comunidades (OREALC/UNESCO, 2017). La importancia de este planteamiento es que para los pueblos indígenas contribuye para poder ser capaces de llevar adelante sus derechos y poder ser partícipe de sus sistemas educativos.

Diferentes documentos (UNESCO, 2015; OREALC/UNESCO, 2017) destacan que la incorporación de materias sobre las cosmovisiones potencia la calidad de la educación y enriquece los currículos con prácticas más contextualizadas y sostenibles. Pero esto se complica en la práctica por la brecha entre los dos modelos de conocer occidentales y las otras epistemologías nativas que se generan en el marco de relaciones de poder y de desigualdad histórica (Lander, 2000). Cabe recordar el texto de la UNESCO (2015) titulado Replantear la educación ¿Hacia un bien común mundial? En este texto se describe la importancia de tomar en cuenta la diversidad como un recurso y en donde se pone énfasis en la exploración de enfoques alternativos a la concepción hegemónica de desarrollo. Estos modelos alternativos están representados por el sumak kawsay o "Buen vivir" como una forma de integrar saberes que permitan establecer otra manera de relacionarse con la naturaleza y otra forma de vínculos sociales y comunitarios.

\section{Educación intercultural bilingüe}

La modalidad en EIB resulta una opción de la educación común cuando las particularidades contextuales lo requieran, pero no obliga ni alberga a todos los niveles educativos. Es decir, está planteada como excepción y no representa a toda la población ni todo el territorio, y cubrir todos los niveles es solo una posibilidad. Luis Enrique López (2006) agrega que varios programas de EIB de la región ostentan la "condición de modalidad educativa, junto a otras formas alternativas que los sistemas educativos tienen para atender de manera remedial y compensatoria a otras poblaciones en riesgo educativo, como podrían ser, por ejemplo, las modalidades de atención a educandos discapacitados o jóvenes y adultos que no asisten al sistema educativo regular" (López, 2006: 2-3).

Finalmente, la EIB representa el modelo institucional con que los Estados de la región garantizan la educación en la lengua y en la cultura del pueblo en cuestión. Cabe agregar que la EIB es la educación que los pueblos reclaman para sí. Ruth Moya (2009), en relación a los pueblos indígenas afirma que, si bien estos reclaman su derecho a educarse en sus propias lenguas y a ampliar las fronteras del bilingüismo, la educación intercultural o la educación con enfoque intercultural no tiene por qué subsumir a la EIB. Por ende, si lo que se pretende es un enfoque de educación intercultural, "el Sistema Educativo no puede eludir la pregunta sobre qué acciones específicas están dirigidas al componente blanco-mestizo para valorar la cultura indígena (también poblaciones afrodescendientes) y reconocerla en igualdad de condiciones" que la cultura dominante (Corbetta, 2016: 42). La etnoeducación tiene este desafío en varios países como Colombia. El concepto de la intercultura- 
lidad nace en América Latina específicamente en la esfera educativa.

Generalmente se atribuye a los lingüistas y antropólogos venezolanos Esteban Mosonyi y Omar González la primera definición del concepto a mediados de los años setenta. Estos se enfocaron específicamente en las experiencias educativas que realizaron con indígenas de los pueblos arahuacos en Venezuela. Dentro de los pioneros de estos estudios se puede encontrar a Vera María Ferrão Candau (2013).

En esta primera fase se encuentra un movimiento de educación escolar indígena, que abraza un conglomerado de experiencias alternativas de educación ejercidas a partir de liderazgos comunitarios relacionados con universidades y sectores progresistas de la iglesia católica. Paralelamente se configuraron materiales didácticos alternativos. Por un lado, la "integración" de estos pueblos indígenas era lo relevante, reconociéndose como importante el derecho a potenciar la cultura de los pueblos. El bilingüismo más que un instrumento de civilización fortalece a los grupos étnicos como una instancia que va más allá de la interculturalidad y presiona que no solo se incluya distintas lenguas sino también culturas al sistema escolar.

En los años ochenta, las luchas indígenas configuran una identidad común que alcanzan un reconocimiento internacional. Por ello, en varios países nace la exigencia de incluir profesores indígenas en sus cargos además administrativos.
Su aporte dentro de la educación intercultural fue denunciar las diversas maneras de discriminación racial, la ideología del mestizaje (Ecuador) y de la "democracia racial" (Brasil). Estas corrientes se basan en que las relaciones pasadas y actuales entre diferentes grupos son de "cordialidad" y que todavía están muy presentes en los imaginarios sociales y las sociedades de América Latina. Los movimientos afrodescendientes también dilucidaron una lectura alternativa de los procesos históricos sobre la participación de las personas afrodescendientes en las guerras y en la formación de los Estados nacionales.

Estos plantearon demandas por reparación ante daños generados por regímenes esclavistas y políticas explícitas o tácitas de blanqueamiento de las poblaciones afrodescendientes. Generalmente partieron sus posturas de producción de conocimiento crítico para la elaboración de contenidos y políticas educativas. Paralelamente las poblaciones afrodescendientes partieron con una demanda de propuestas de políticas en la valorización de las identidades culturales afrodescendientes, además de la inclusión en los currículos escolares y en los materiales pedagógicos de componentes propios de esas cosmovisiones y sobre los procesos históricos de resistencia vividas por estas. Luego encontramos la etapa en donde Ferrão Candau (2013) menciona los aportes a la educación intercultural que se realizó en el continente latinoamericano con las experiencias de la educación popular a partir de los desarrollos por Paulo Freire. 
Recordemos que desde la década de los setenta estas experiencias privilegiaron los espacios de educación no formal, pero luego ejercieron influencia sobre los sistemas educativos formales, específicamente hacia el final de los años ochenta y principios de los años noventa. Es decir, desde la filosofía los términos de la interculturalidad y cultura permiten entender los dilemas que presenta la comprensión del concepto.

Desde un punto de vista pedagógico, lo intercultural (Abdallah-Pretcheille 1992, 1999) puede abarcar diferentes áreas de estudio. Aquí es necesario referirse a dos plataformas de comprensión: primero, como orientación de problemas que tienen relación con la enseñanza; y en segundo lugar, la dimensión cultural asociada con todo aprendizaje. Es importante hacer presente que en América Latina los conceptos de interculturalidad y multiculturalidad generalmente se relacionan con la educación intercultural bilingüe. Esta comprensión nace a partir de las demandas de dirigentes indígenas e intelectuales de las ciencias sociales para contextualizar los conocimientos escolares con los saberes culturales de los pueblos indígenas. En este sentido, el concepto intercultural está determinado por saberes que surgen de relaciones interpersonales e intergrupales.

Sin embargo, las instituciones gubernamentales se manejan generalmente a partir de una visión monocultural, donde lo intercultural pertenece solo a los indígenas. Esta forma de comprender la interculturalidad nace de la idea del multi- culturalismo, en donde las políticas públicas no integran a las comunidades indígenas al país, por lo cual son segregadas de manera sistemática (Fernández, 2003). Paulo Freire afirma que existen algunos saberes primordiales para la práctica docente de educadores/as críticos y progresistas y que son también inherentes a los/ las educadores/as interculturales.

Cabe recordar uno de los argumentos desarrollados por Freire que representa el pilar de su pensamiento: “[...] quien está formando, desde el principio mismo de su experiencia formadora, al asumirse también como sujeto de la producción del saber, se convenza definitivamente de que enseñar no es transferir conocimiento, sino crear las posibilidades de su producción o de su construcción" (Freire, 1997: 24). Es decir, quien enseña aprende al enseñar y quien aprende enseña al aprender.

En un cuarto ámbito, fue relevante la relación entre interculturalidad y educación por parte de un grupo importante de países de la región, con su carácter multiétnico, plurilingüe y multicultural en sus constituciones nacionales durante las décadas de 1990 y 2000 . Dentro de este contexto encontramos a 11 países contando a la Argentina, el Estado Plurinacional de Bolivia, el Brasil, Colombia, el Ecuador, Guatemala, México, Nicaragua, el Paraguay, el Perú y la República Bolivariana de Venezuela. Cabe recordar que tal reconocimiento tuvo consecuencias en las reformas educativas que consideraron de alguna forma la esfera intercultural. Es decir, rela- 
cionando los contenidos curriculares o introduciendo de forma transversal temas en relación a la diferencia cultural.

Si bien estas reformas significan grandes avances, no han estado exentas de ambigüedades en la medida que la mayoría de los gobiernos de estos países llevaron adelante políticas neoliberales que en materia social estuvieron caracterizadas por su corte focalizado y compensatorio. Dentro de este punto cabe recordar a Félix Pazi, primer Ministro de Educación indígena del Estado Plurinacional de Bolivia, quien denuncia que no es conveniente enfrentar un proceso de diálogo intercultural desde la complicada situación en la que se encuentran los pueblos indígenas en relación a la cultura hegemónica.

Otra corriente argumenta que la intra e interculturalidad deben ser corrientes paralelas que se complementan dialécticamente. Luego, para las poblaciones afrodescendientes como Ecuador y Colombia se plantean dos conceptos que desde la etnoeducación se fusionan o son equivalentes al de intraculturalidad e interculturalidad. Es decir, la educación apuntalada al autorreconocimiento (la educación para sí) y la educación relacionada al reconocimiento de los otros grupos étnicos (no afrodescendientes e indígenas) (SITEAL, 2011).

Cabe recordar que la lucha por el autorreconocimiento (lucha de puertas hacia adentro) es comprendida como la reconstrucción de la memoria desde el pensamiento cimarrón (Walsh y
León, 2007). Es decir, cobra valor para el presente como una recuperación de la historia que fue negada por el sector no afro descendiente y por el componente indígena. La lucha por el reconocimiento de la cultura afrodescendiente (lucha de puertas hacia afuera) demanda obtener un reconocimiento de las personas no afrodescendientes e indígenas (SITEAL, 2011).

Por conclusión, cabe reflexionar más a fondo la propia formación y el desconocimiento de las culturas indígenas y afrodescendientes para transformar los modelos pedagógicos hegemónicos. Es decir, cabe ser autocríticos de las identidades docentes que dichos modelos han formado (Navarro, 2004). Por ello, es necesario reconocer para la EIB la diversidad indígena entre si y en interacción con las poblaciones afrodescendientes (López, 2006). Otro punto relevante es profundizar en los saberes indígenas, es decir, el "buen vivir" y sus cosmovisiones afrodescendientes para incluir a estas en las políticas educativas. (Requejo, 2001).

El desafío relevante dentro de este contexto debería enfocarse en la educación superior en EIB y en la formación de los docentes (Navaro, 2004; SITEAL, 2011). Para ello, es importante que los docentes dominen contenidos de relación entre lengua, cultura y sociedad, además de que los docentes indígenas y no indígenas dominen metodologías estratégicas para incluir a miembros de las comunidades en procesos participativos. En la esfera practica pedagógica es clave el uso de las lenguas (lengua materna y lengua domi- 
nante) como un objeto de estudio o vehículo de enseñanza (Gualdieri, 2004; López, 2006). Un tema también relevante consiste en incluir la perspectiva de género y la valoración de la mujer en las prácticas pedagógicas, en la recreación de las culturas indígenas y afodescendientes y en las políticas.

Respecto de la demanda a la Educación Intercultural Bilingüe contextualizada en relación con los pueblos indígenas y la población afrodescendientes, especialmente las provenientes de las experiencias de cimarrones, palenqueros o de las quilombolas, se enmarcan dentro de luchas políticas que ya tienen su historia. Es decir, el territorio indígena, por ejemplo, configura el punto de partida de las demandas hacia el Estado porque este compone la base en donde se insertan sus propias cosmovisiones en relación al espacio geográfico, social y simbólico. En síntesis, una educación debe albergar las características e intereses de los pueblos para reconocer, mantener y desarrollar la lengua, la cultura y la territorialidad de cada uno de estos.

\section{Pedagogía intercultural etnomatemática}

A continuación se analizará el aporte de la pedagogía etnomatemática para contextualizar y revitalizar la educación intercultural tomando en cuenta otras cosmovisiones. Recordemos que la experiencia misma del tiempo como tal entre los pueblos primitivos no equivale siempre a la experiencia del tiempo de un occidental moderno. Paralelamente, algunos autores plantean la idea de que la visión de que las poblaciones indígenas de México y la concepción indígena mapuche se manejan en un tiempo circular. Esta es una construcción colonial, ya que, en el caso de los mayas, por ejemplo, articulan la circularidad con la linealidad, es decir, proponen una construcción híbrida del tiempo.

Esa propuesta resulta entendible como una suerte de espiral que se desarrolla simultáneamente en la circularidad, pero que avanza de forma progresiva. Es decir, este proceso se puede comprender a partir de un 'modelo de resorte'. Por otra parte, tenemos la concepción judeo-cristiana del tiempo, desgarrada también entre linealidad y circularidad: el regreso del Mesías y el final de los tiempos con el Apocalipsis indican una combinación de tiempo lineal y circular.

Pero volviendo al tema de nuestra investigación trataremos a continuación también la concepción mapuche de tiempo. Para ello, es necesario entrar en la concepción de marcas o señales temporales (el día y las estaciones del año) aplicada en la cotidianeidad pero también en sus espacios educativos formales. Recordemos que las categorías principales son el xipantu, descrito como ciclo de tiempo anual, y el antü, ciclo del día. Estos dos conceptos son utilizados en la enseñanza por los kimches, quienes recurren a la memoria social mapuche de padres y abuelos que forman parte en la educación familiar. Quilaqueo (2002) afirma que los kimches son los portadores del saber cultural y social mapuche, es decir, educadores que han enseñado históricamente a los 
jóvenes las categorías de tiempo y espacio.

Cabe agregar que la descripción de tiempo y espacio en la cultura mapuche tiene rasgos distintos de las concepciones occidentales. Es decir, para las nociones mapuches de tiempo en el discurso educativo y específicamente de los kimches, éstas se establecen para las etapas del día y el año: el día como antü y el año como xipantu. Es así que parten de rasgos propios para reconocer el transcurso del tiempo que están relacionados con la naturaleza, como por ejemplo, en la observación del sol, su rotación y su mudanza por los cerros. Pero la diferencia en la noción de tiempo entre los kimches de las territorialidades pewenche y wijuche surge por las características geográficas. O sea, va a depender de las características contextuales (territoriales) en relación con el territorio geográfico, como por ejemplo en la observación de cerros.

Por otro lado, los kimches coinciden en la idea de que ambas territorialidades se trata al sol como un elemento importante, construyendo una relación entre la observación de la naturaleza, el recorrido del sol y el desenvolvimiento de la agricultura. Por ello, se afirma que los elementos más relevantes respecto del discurso de los kimches se relacionan con el día y con el año. Estos saberes del discurso educativo de los kimches están relacionados con la concepción del tiempo y deberían incorporarse al currículum escolar, pero específicamente al subsector de Comprensión del Medio Natural, Social y Cultural y al subsector de educación matemática, por- que ambos subsectores trabajan en la actualidad la noción solo occidental, pero sin considerar que la lógica del tiempo en un contexto mapuche es diferente.

Por ello, es necesario que las mallas curriculares universitarias en la educación inicial dispongan también de una pedagogía intercultural etnomatemática que incluyan estas categorías de espacio y tiempo, y así poder, mediante una educación intercultural con un cambio curricular, incorporar en parte esta cosmovisión mapuche y su concepción de tiempo en las escuelas que se encuentran en un contexto mayoritariamente indígena o mapuche. En síntesis, es posible superar la distancia epistemológica entre el conocimiento escolar y los conocimientos propios de los mapuche o no mapuche por medio de espacios amables de dialogo que incorpore elementos presentes entre ambas racionalidades (mapuche y científico occidental).

Finalmente cabe agregar que en Colombia, lugar donde existen varias cosmovisiones, la Universidad de Antioquía ha logrado avanzar ejemplarmente con respecto a estas materias. Dentro de sus carreras universitarias existe un nuevo modelo pedagógico enfocado en la búsqueda de alternativas de formación universitaria indígena, distintas a la de admisión especial en programas regulares, reconociendo la importancia y obligatoriedad del trabajo en equipo, directo con una organización indígena y construyendo conjuntamente con ésta instrumentos sustantivos de mejora de su vida comunitaria. 


\subsection{Licenciatura en Pedagogía de la Madre Tie- rra de la Universidad de Antioquía}

En la Universidad de Antioquía en Medellín existe la licenciatura en Pedagogía de la Madre Tierra en Colombia como un programa específico e innovador de educación superior y especial en su género en América Latina. Este pretende profundizar en la problemática de la educación intercultural universitaria adaptada a las necesidades y especificidades de las comunidades indígenas. Paralelamente es una licenciatura que trata, desde diversas dimensiones y ámbitos interconectados, con el desarrollo de los planes de vida indígena y las cosmovisiones y cosmogonías de sus pueblos, el fomento del diálogo intercultural y la diversidad cultural.

Esta carrera de licenciatura de la Universidad de Antioquía es un buen ejemplo de cómo se puede contextualizar la educación intercultural y etnomatemática, es decir, adaptándola como base curricular en universidades chilenas o latinoamericanas para incluir el concepto de tiempo de los indígenas o kimches $\mathrm{u}$ otras cosmovisiones autóctonas, por medio de un auténtico “diálogo de saberes".

Cabe resaltar que en la mayoría de los programas académicos universitarios, los pensamientos (cosmovisiones) y saberes indígenas generalmente son negados, invisibilizados o desconocidos, resultando como consecuencia un contexto académico en el que se ha posi- cionado el conocimiento científico occidental como el único "verdadero", bajo las premisas de "universalidad" y "neutralidad", acabando por deslegitimar saberes procedentes de entornos culturales diferentes.

Es decir, la investigación sobre estas experiencias es todavía insuficiente a pesar de que cada vez se conciben más relevantes en Latinoamérica. Daniel Mato (2008) afirma que el Instituto Internacional de la Unesco para la Educación Superior en América Latina y el Caribe ha concebido 50 instituciones de educación superior dedicadas a las comunidades indígenas y afrodescendientes, ya sea parcial o totalmente, con diferencias significativas en sus concepciones. A su vez, en el caso de Colombia, con excepción de algunos programas de etnoeducación, hasta la creación de la Licenciatura en Pedagogía de la Madre Tierra (LPMT) de la Universidad de Antioquía, no se había contado con modelos pedagógicos específicos que tuvieran en cuenta las particularidades culturales de los estudiantes indígenas y las necesidades, cosmovisiones y sistemas de conocimiento tradicional de sus comunidades.

Por otro lado, es esencial reconocer los contextos previos de los modelos educativos superiores de las comunidades indígenas. Para ello, diversas iniciativas de educación superior orientadas hacia los pueblos originarios han venido siendo difundidas y apoyadas a través de eventos mundiales e instituciones como la Asociación Mundial de Educación Superior de los Pueblos Indí- 
genas (winhec) o la Conferencia en Educación de los Pueblos Indígenas del Mundo (wipce). Pero también la presencia de organismos y agencias de cooperación internacional o bilateral, de fundaciones y de universidades extranjeras en el ámbito de la educación indígena en el continente americano se ha incrementado considerablemente en la última década. Otras iniciativas importantes para desarrollar programas de educación superior indígena (Mato, 2011: 63-85) que van desde marcos asimilativos y convencionales son las universidades interculturales estatales en México (Rebolledo, 2005).

Paralelamente, otros centros de investigación están más centrados en las cosmovisiones indígenas, como la Universidad de la Tierra en Chiapas, México, el Programa de Educación Intercultural Bilingüe en Cochabamba, Bolivia, que ofrece una maestría en este campo, o la Universidad Intercultural de los Pueblos Indígenas "Amawtay Wasi" en Ecuador. En Colombia, producto del surgimiento de las luchas indígenas, la recuperación de tierras y el reconocimiento de su autonomía, el Estado ha ido adaptando lentamente sus organizaciones como interlocutoras, dando paso a cambios legislativos de reconocimiento de sus demandas. A partir de todo ello, ha nacido la apertura de licenciaturas en Etno-educación en algunas universidades de Colombia para la formación de los docentes indígenas, con experiencias a resaltar por el diálogo que se construye entre equipos universitarios y organizaciones indígenas.
Sin embargo, por la rigidez de las estructuras universitarias existen varias organizaciones que han apostado por sus propias propuestas de educación superior con programas no reconocidos por el Ministerio de Educación Nacional, como es el caso de la Universidad Autónoma Indígena Intercultural (UAII), del Consejo Regional Indígena del Cauca (CRIC). Cabe resaltar que la educación propia adaptada a las características socioculturales indígenas en Colombia no se abordó realmente hasta la década de los ochenta, si bien acabó impulsándose definitivamente con la promulgación de la Constitución Política de Colombia de 1991, que produjo una significativa apertura en el Estado.

Durante años, la sociedad colombiana se ha preocupado por el reconocimiento de los derechos indígenas, sus lenguas y su cultura. Recordemos que un mandato constitucional se plasmó reglamentariamente en la Ley 115 General de Educación, la cual orienta al Estado a implementar programas educativos especializados para todos y cada uno de los grupos étnicos de Colombia. Luego el Decreto 804 de 1995 extendió los enfoques y planteamientos de la educación para grupos étnicos, en particular en lo que se refiere a los principios de integralidad, diversidad lingüística, autonomía, participación comunitaria, interculturalidad, flexibilidad, progresividad y solidaridad, asumiendo el gobierno la financiación de los proyectos etno-educativos.

La implicación directa del Estado facilitó desde entonces la oferta por parte de universidades 
públicas y privadas de cupos, becas e incluso programas especiales para estudiantes indígenas. Por ello, en la actualidad existen carreras de Etno-educación en un número significativo de universidades del país y se han puesto en marcha programas de especialización y maestría en esta temática, así como de doctorado en educación multicultural y educación para grupos étnicos.

Por otro lado, varias organizaciones indígenas regionales, por sí mismas o en alianza con universidades oficiales, también han ido introduciendo sus propios programas de educación especializada en las temáticas más pertinentes a su movimiento social e incluso algunos indígenas han conseguido impartir clases de lenguas y otras temáticas indígenas en varias universidades oficiales. Es de destacar, en este sentido, el Instituto Departamental para la Educación Indígena (INDEI) de la Organización Indígena de Antioquia (OIA), máxima autoridad de los pueblos indígenas del Departamento, que puso en marcha en 2003 su denominado "Proyecto Etnoeducativo Comunitario (PEC)", el cual establece la necesidad de la formación post-secundaria de sus líderes y educadores, en colaboración con instituciones de educación superior.

La Universidad de Antioquía, mediante la firma de un Convenio Marco de Cooperación en 2004 con la OIA, renovado después en 2009 , dio paso a la creación del denominado Programa de Educación Indígena, que para su coordinación abrió por primera vez en su historia una plaza docente para un profesor indígena. Cabe resaltar que al comienzo, la idea de licenciatura estaba dirigida a los pueblos indígenas de Antioquía. Luego, se concretó la propuesta de que esta también se pueda ofrecer en el futuro a líderes, educadores y bachilleres de otros pueblos indígenas del país (o personas no indígenas comprometidas), pero mediante la adecuación de contenidos de acuerdo con cada contexto y en concertación con las organizaciones o comunidades solicitantes.

Por otro lado, las maestras y maestros de lenguaje tendrían que cuestionar su figura de técnicos que determinan lo incorrecto y lo correcto a partir del anquilosado ideal de un lenguaje "culto", como si en la cotidianidad simbólica y comunicativa de los distintos lenguajes no se produjera constantemente cultura. Es decir, en vez del rol de técnicas y técnicos de la prescripción lingüística, habría que reflexionar sobre la dimensión política del lenguaje y su papel en los circuitos ideológicos y de poder (Giroux, 1990). Es decir, a partir de las metodologías propias con las cuales las sociedades indígenas reproducen sus conocimientos y valores a las nuevas generaciones, la LPMT intenta precisamente rescatar un lugar para ellas en la enseñanza de la lengua materna y del castellano, en tanto que ellas mismas ya trazan rutas pedagógicas.

Dentro del Núcleo Básico se pueden apreciar tres ejes temáticos que orientan horizontalmente sus contenidos: 1) reencuentro con los saberes y realidades indígenas; 2) diálogo con los "otros"; y 3) el futuro de las comunidades indígenas. Es- 
tos ejes temáticos se desarrollan, asimismo, a través de los siguientes ejes verticales: 1) vida de los pueblos indígenas y diálogo de saberes; 2) formación pedagógica desde la interculturalidad. Por su parte, el Núcleo de Énfasis se estructura en cuatro ejes temáticos: 1) el territorio como la "piel" de la Madre Tierra; 2) el agua como deidad de la Madre Tierra; 3) el mundo de las plantas como seres de la Madre Tierra; y 4) el mundo de los animales como seres de la Madre Tierra.

El objetivo académico de formación pedagógica desde la interculturalidad apunta a la necesidad de ser conscientes de los prejuicios históricamente creados y explorar maneras más equitativas y creativas de interaccionar pueblos y saberes. Es decir, el rol pedagógico de la LPMT parte por ello de un ejercicio de "descolonización" de lo que son y piensan los pueblos indígenas, preguntándose por cuestiones como: ¿Quiénes son, de dónde vienen, cuáles son sus intereses y necesidades, Qué sueñan? En otras palabras, se parte de la interculturalidad desde la reflexión pedagógica.

Conceptualmente no se parte solo de una posición teórica o un diálogo de y/o entre culturas, sino como una actitud o postura que debemos asumir las personas para aprender a convivir con los "otros" y para vivir las referencias identitarias propias en relación con las de 'otros', es decir, identificar aquellas limitaciones propias, teóricas y prácticas, que nos dirigen a considerar la cultura "propia" como la única verdadera (Fornet-Betancourt, 2004). Dentro de su conte- nido se encuentran los siguientes seminarios: 1) Núcleo Básico: Etnomatemáticas y Seminarios de educación, lenguajes y comunicación; y 2) Núcleo de énfasis: Etnomatemáticas electivas y Pedagogías desde la Diversidad Cultural. D’Ambrosio (2001), entre otros autores, señala que la Etnomatematica es una propuesta de carácter filosófico que viene siendo discutida desde la década de los años ochenta por numerosos autores que han puesto en debate la producción, validación y legitimación del conocimiento matemático en las diferentes prácticas sociales.

En la LPMT, la Etnomatemática busca centrar su atención en la comprensión y problemática de la producción y construcción del conocimiento matemático en las diferentes comunidades indígenas, como resultado de la interpretación del mundo, de la dialéctica ser humano-naturaleza y su legitimación desde y para unas prácticas sociales y culturales propias de cada comunidad. La Etnomatemática se configura desde las dimensiones conceptual, histórica, cognitiva, epistemológica, política y educativa (D’Ambrosio, 2001), que permiten considerar al conocimiento matemático como una producción cultural y social de los diferentes grupos y comunidades.

El objetivo es denunciar y transformar las relaciones de poder que conducen los procesos de validación y legitimación del saber; y en lo relacionado con el proceso pedagógico, el desafío está centrado en las posibilidades y estrategias de enseñanza y de aprendizaje que consideren 
el ambiente multicultural del aula (Monteiro et al., 2004).

Dentro de este contexto, los docentes y estudiantes de la LPMT se constituyen en protagonistas y desencadenadores de variadas posibilidades de procesos de enseñanza y de aprendizaje, considerando sus contextos socioculturales específicos. Es decir, se elabora un espacio para comprender y reflexionar las relaciones intra e interculturales presentes en diferentes realidades, permitiendo que se reconozcan e incorporen al currículo de la escuela, prácticas y conocimientos producidos fuera del contexto escolar (López y Prada, 2004). La Etnomatemática permite comprender diferentes grupos culturales que construyen el conocimiento matemático, así como las maneras del saber/ hacer matemático de una cultura (Jaramillo, 2006). Para ello, la LPMT ha incluido el reconocimiento y fortalecimiento permanente de las lenguas maternas, así como la recontextualización de la lengua castellana desde el pensamiento indígena para evidenciar en procesos de enseñanza y aprendizaje el saber cultural, tradicional y cosmogónico de los pueblos indígenas. La LPMT asume que no solo trata de estudiar las lenguas y describir sus estructuras, sino de reconocer e interpretar los significados de vida en ellas inmersos, en complejas interacciones de los diversos lenguajes de las comunidades en ámbitos históricos, políticos, económicos, sociales y culturales.

Dentro de este contexto, la investigación de-colonial manifiesta una búsqueda de alternativas para saber y ser, contribuyendo así a la preservación de la Madre Tierra y los seres que la habitan. Cabe resaltar que la Universidad de Antioquía ha avanzado fantásticamente con esta licenciatura en la conformación de un nuevo modelo pedagógico enfocado a la búsqueda de alternativas de formación universitaria indígena distintas a la de admisión especial en programas regulares. Paralelamente, siempre reconociendo la importancia y obligatoriedad del trabajo en equipo y directo con una organización indígena y construyendo conjuntamente con ésta instrumentos sustantivos de mejora de su vida comunitaria.

Cabe mencionar que este programa de educación superior puede considerarse una de las pocas experiencias en el mundo de estas características al tener, sobre todo, dos rasgos esenciales: 1) un nuevo modelo pedagógico con una construcción colectiva propia, colaborativa y participativa, por medio de los agentes implicados en el proceso formativo. Es decir, con los mismos estudiantes y los maestros, además de articular esfuerzos e iniciativas de una organización indígena de Antioquía y una universidad pública oficial estatal; y 2) la combinación de perspectivas críticas, reflexivas, creativas, de-coloniales, de liderazgo colectivo y de género.

Todo ello abarca el desarrollo de metodologías formativas interculturales que tienen a la "Madre Tierra" como eje de conocimiento y aprendizaje integral, así como la adopción del Seminario Pedagógico, que ha facilitado la valoración de la cultura propia y de las potencialidades ins- 
titucionales.

Por ello, cabe preguntarse cómo debería ser el itinerario de una pedagogía que incluya estos conceptos de espacio y tiempo de los indígenas, mapuche $u$ otras cosmovisiones partiendo de la interculturalidad contextualizada. La interculturalidad desde la reflexión pedagógica se manifiesta no como una posición teórica o un diálogo de y/o entre culturas, sino también como una postura que debemos asumir para aprender a convivir con los "otros", vivir las referencias identitarias propias en relación con las de 'otros' e identificar aquellas limitaciones propias, teóricas y prácticas, que nos llevan a considerar la cultura "propia" como la única verdadera (Fornet-Betancourt, 2004). Dentro de estos pilares nos detendremos en la Etnomatemática, que es una propuesta de carácter filosófico que viene siendo discutida desde la década de los años ochenta por numerosos autores ( $D^{\prime}$ Ambrosio, 2001; Lizcano, 2002; Knijnik, 2003; Monteiro, 2004). Estos investigadores han puesto en debate la legitimación del conocimiento matemático en las diferentes prácticas sociales.

La Etnomatemática busca comprender la problemática de la producción y construcción del conocimiento matemático en las diferentes comunidades indígenas como resultado de la interpretación del mundo, de la dialéctica ser humano-naturaleza y de lógicas culturales propias de cada comunidad. La Etnomatemática se elabora desde los ámbitos conceptuales, históricos, cognitivos, epistemológicos, políticos y educa- tivos (D’Ambrosio, 2001), los cuales permiten comprender al conocimiento matemático como una producción cultural y social de los diferentes grupos y comunidades.

Además, permite comprender dimensiones que permiten reconocer la matemática bajo una perspectiva de la investigación y que tiene un fin en sí misma. Desde los docentes y estudiantes, este modelo concibe a estos como protagonistas y desencadenadores de variadas posibilidades de procesos de enseñanza y de aprendizaje, pero siempre comprendiendo sus contextos socioculturales específicos. Es así como dentro de estas relaciones intra e interculturales presentes se busca comprender las diferentes realidades y contextos, permitiendo que se reconozcan e incorporen al currículo de la escuela, prácticas y conocimientos producidos fuera del contexto escolar (López y Prada, 2004).

La Etnomatemática permite conocer cómo los diversos grupos culturales construyen el conocimiento matemático, así como las formas del saber/ hacer matemático de una cultura. Respecto a los lenguajes e interculturalidad, se busca formar maestros y maestras que, desde el conocimiento profundo del rol de los lenguajes y la lengua en la perseverancia de las comunidades ancestrales, elaboren procesos pedagógicos en la comunidad y la escuela desde una óptica crítica y creativa. La pedagogía intercultural ha considerado importante el reconocimiento y fortalecimiento continúo de las lenguas maternas y la 
recontextualización de la lengua castellana desde el pensamiento indígena, con el fin de evidenciar en los procesos de enseñanza y aprendizaje el saber cultural, tradicional y cosmogónico de los pueblos indígenas.

Dentro de la pedagogía intercultural etno-matemática no solo se trata de estudiar únicamente las lenguas y describir sus estructuras, sino también de reconocer e interpretar los significados de vida en ellas inmersos. Es decir, la compleja interacción de los diversos lenguajes de las comunidades con asuntos históricos, políticos, económicos, sociales y culturales. Solo partiendo de este reconocimiento identitario con la lengua y la cultura materna es posible establecer un diálogo intercultural con la lengua castellana $u$ otras lenguas, que sea de carácter interactivo y creativo, y no de sumisión, des-legitimación y pérdida de las lenguas originarias.

Este currículo de la Educación Intercultural Bilingüe también busca encontrar posibilidades de aportar a un cambio social. Desde esta concepción, se mantiene lo originario, pero también se tiene el interés por la otra cultura y a su vez se cree que esta intención de respeto cultural se acomoda voluntariamente como consecuencia de intereses específicos (Demorgen y Lipiansky, 1999).

La interculturalidad solo puede existir en contexto donde las culturas se estiman iguales, sin serlo o de una forma más bien parcial. Sin embargo, cabe recordar que el multiculturalismo, la multiculturalidad como la interculturalidad son conceptos inseparables de su cultura o contexto situado de la cual emergen como un conocimiento estratégico que representan ideas del pasado o del presente de sus sociedades. Esto se percibe claramente en la dinámica natural que se refleja en ciertas características provenientes de países coloniales que poseen concepciones totalmente opuestas a las de los indígenas.

El término interculturalidad abarca varias áreas comprensivas como la dinámica de su origen que se basa en dos aspectos principales: en lo epistemológico y en lo histórico. En primer lugar, se debe relacionar lo intercultural con una institución social, pero tomando en cuenta que no se define la interculturalidad per se, como por ejemplo en la economía. Dentro de sus características hay que considerar elementos tales como lo religioso y lo político, entre otros ámbitos. Por otro lado, se debe tener presente que lo intercultural se encuentra dentro de un contexto histórico en curso. El tercer punto relevante se relaciona con el surgimiento de la interculturalidad que nace a partir de corrientes anteriores, las cuales desembocan en algo nuevo. Sin embargo, dentro de esta dinámica las grandes culturas históricas van a tener siempre mayor influencia.

Es importante hacer presente que en América Latina los conceptos de interculturalidad y multiculturalidad generalmente se relacionan con la educación intercultural bilingüe. Esta comprensión nace a partir de las demandas de diri- 
gentes indígenas e intelectuales de las ciencias sociales para contextualizar los conocimientos escolares con los saberes culturales de los pueblos indígenas (Quilaqueo et al., 2005).

Esta forma de comprender la interculturalidad nace de la idea del multiculturalismo en donde las políticas públicas no integran a las comunidades indígenas al país, por lo cual son segregadas de manera sistemática (Fernández, 2003).

\section{Conclusión}

Cabe concluir que muchas de estas corrientes de la educación intercultural se encuentran en constante tensión por tratarse de relaciones pasadas y actuales entre diferentes grupos que todavía están muy presentes en los imaginarios sociales de las sociedades de América Latina, como sucede por ejemplo con los movimientos afrodescendientes, quienes a su vez interpretan su historia por medio de una lectura alternativa de los procesos históricos sobre la participación de las personas afrodescendientes en las guerras y en la formación de los Estados nacionales.

Además, estos movimientos solicitaron generalmente demandas por reparación ante daños generados por regímenes esclavistas y políticas explícitas o tácitas de blanqueamiento de las poblaciones afrodescendientes. Paralelamente sus posturas de producción de conocimiento crítico para la elaboración de contenidos y políticas educativas partieron por una demanda de propuestas de políticas en la valorización de las identidades culturales afrodescendientes.

Con esto exigían la inclusión en los currículos escolares y en los materiales pedagógicos los componentes propios de sus cosmovisiones sobre los procesos históricos de resistencia vividas por las poblaciones afrodescendientes. Ferrão Candau (2013) menciona los aportes a la educación intercultural que se realizó en el continente latinoamericano con las experiencias de la educación popular a partir de los desarrollos por Paulo Freire. Recordemos que desde la década de los setenta estas experiencias privilegiaron los espacios de educación no formal, pero luego ejercieron influencia sobre los sistemas educativos formales, específicamente hacia el final de los años ochenta y principios de los años noventa. Paralelamente es necesario referirse a dos plataformas de comprensión: primero como orientación de problemas que tienen relación con la enseñanza; y la dimensión cultural asociada con todo aprendizaje. Es decir, cabe resaltar la importancia de hacer presente que en América Latina los conceptos de interculturalidad y multiculturalidad generalmente se relacionan con la educación intercultural bilingüe.

Sin embargo, la tensión nace porque la mayoría de las instituciones gubernamentales latinoamericanas en su práctica pedagógica se manejan generalmente a partir de una visión monocultural, donde lo intercultural pertenece solo a los indígenas. Esta forma de comprender la interculturalidad nace de la idea del multiculturalismo en donde las políticas públicas no integran 
a las comunidades indígenas al país por lo cual son segregadas de manera sistemática (Fernández, 2003).

Luego fue relevante la relación entre interculturalidad y educación por parte de un grupo importante de naciones de la región con su carácter multiétnico, plurilingüe y multicultural en sus constituciones nacionales durante las décadas de 1990 y 2000, como por ejemplo, 11 países contando a la Argentina, el Estado Plurinacional de Bolivia, el Brasil, Colombia, el Ecuador, Guatemala, México, Nicaragua, el Paraguay, el Perú y la República Bolivariana de Venezuela.

Cabe recordar que tal reconocimiento tuvo consecuencias en las reformas educativas que consideraron de alguna forma la esfera intercultural, es decir, relacionando los contenidos curriculares o introduciendo de forma transversal temas en relación a la diferencia cultural. Si bien estas reformas significan grandes avances, no han estado exentas de ambigüedades en la medida que la mayoría de los gobiernos de estos países llevaron adelante políticas neoliberales que en materia social estuvieron caracterizadas por su corte focalizado y compensatorio.

Dentro de este contexto cabe recordar a Félix Pazi, primer Ministro de Educación indígena del Estado Plurinacional de Bolivia, quien denuncia que no es conveniente enfrentar un proceso de diálogo intercultural desde la situación complicada en la que se encuentran los pueblos indígenas en relación a la cultura hegemónica.
Además agrega que primero es necesario valorar la propia cultura, costumbre y la lengua para revertir las creencias que se han mantenido desde el colonialismo.

Dentro de este pensamiento se argumenta que la intra e interculturalidad deben ser corrientes paralelas que se complementan dialécticamente. Luego para las poblaciones afrodescendientes de países como Ecuador y Colombia se plantean dos conceptos que desde la etnoeducación se fusionan o son equivalentes al de intraculturalidad e interculturalidad. Es decir, la educación apuntalada al autorreconocimiento (la educación para sí) y la educación relacionada al reconocimiento de otros grupos étnicos (no afrodescendientes e indígenas) (SITEAL, 2011).

Cabe recordar que la lucha por el autorreconocimiento (lucha de puertas hacia adentro) es comprendida como la reconstrucción de la memoria desde el pensamiento cimarrón (Walsh y León, 2007). Es decir, cobra valor para el presente como una recuperación de la historia que fue negada por el sector no afro descendiente y por el componente indígena. La lucha por el reconocimiento de la cultura afrodescendiente (lucha de puertas hacia afuera) demanda obtener un reconocimiento de las personas no afrodescendientes e indígenas (SITEAL, 2011).

El desafío dentro de este contexto debería enfocarse en la educación superior en EIB y en la formación de los docentes (Navaro, 2004; SITEAL, 2011). Para ello, es importante que los docentes 
dominen contenidos de relación entre lengua, cultura y sociedad, además de que los docentes indígenas y no indígenas dominen metodologías estratégicas para incluir miembros de las comunidades en procesos participativos. En la esfera practica pedagógica es clave el uso de las lenguas (lengua materna y lengua dominante) como un objeto de estudio o vehículo de enseñanza (Gualdieri, 2004; López, 2006).

Sin embargo, paralelamente es necesario proporcionar a este currículum intercultural las herramientas básicas relacionadas con el ámbito valórico. Es decir, la aplicación de este currículum intercultural en los establecimientos escolares (y en algunas de las mallas universitarias) debería estar siempre acompañada por principios valóricos (ética mínima) que darán sustento a este cambio de paradigma.

Es decir, como una propuesta alternativa de solución a todas estas tensiones respecto a la educación intercultural, es importante comprender el alcance de la etnoeducación como propuesta educativa. En Colombia su desarrollo está relacionado con la Cátedra de Estudios Afrocolombianos, el cual representa un avance relevante en materia de reconocimiento de derechos y de la incorporación del aporte cultural afro a la construcción de las naciones, como por ejemplo promover estrategias para trabajar la heterogeneidad de demandas y propuestas al interior de las poblaciones afrodescendientes.

Recordemos el informe de SITEAL (2011), en donde se estudian las demandas en la educación que suponen diferentes perspectivas. La disputa se concentra en los que sostienen una crítica más estructural al sistema educativo y demandan una educación en particular para los propios afrodescendientes, en tanto otros reclaman una educación "para todos" con la incorporación en los currículos de historia y cultura afro.

Por ello, es relevante que los Estados deban propiciar instancias de participación de las propias comunidades para pensar un currículo acorde a las necesidades de cada una de ellas. Paralelamente, pensar los currículos con la incorporación de las epistemes de los propios pueblos indígenas, destacando la relevancia de incorporar otras maneras de conocer no occidentales a los planes de estudio no solo de escuelas con mayoría indígena, sino avanzando mas allá de la modalidad educativa (EIB) con el fin de que tales conocimientos y saberes puedan ser transversales a todo el sistema educativo. Esto amortigua los estereotipos construidos históricamente y promueve la legitimidad como pueblo en tanto productor de propios sistemas de conocimiento.

Por otro lado, se incorporan en los currículos de planes y programas de todos los niveles las problemáticas específicas que afectan a los pueblos indígenas. Recordemos que los problemas históricos que constituyen el eje de las demandas de aquellos son, en general, el derecho a la tierra y al territorio. Las condiciones de precariedad de posesión de tierras son un problema de largo plazo y es necesario concientizar acerca de ello 
en el ámbito educativo. Este punto refuerza la idea de la construcción de políticas educativas con activa participación de los miembros de pueblos indígenas.

Una forma de solucionar estas tensiones en la Educación intercultural es valorizar la pedagogía etnomatemática y sus formas de construir conocimiento. La formación de profesores en la LPMT, considerando la etnomatemática como un elemento central, amplió los horizontes para repensar la Educación Matemática Indígena en las universidades con una perspectiva decolonial e intercultural, porque desde este ámbito se configuran currículos y pedagogías críticas para ver la enseñanza y el aprendizaje de las matemáticas entrelazados a la pregunta ¿A quién enseñamos? Esta centra su mirada en contextos particulares movilizadores de conocimientos matemáticos, esto es, en sujetos específicos, al mismo tiempo que nos aleja de la pregunta ¿Qué enseñar?, centrada única y exclusivamente en el contenido y en una visión disciplinar del conocimiento, tal como la escuela moderna se ha organizado.

La LPMT es un ejemplo en la construcción de programas de formación más apropiados para los pueblos indígenas. Además, surge un programa de formación docente donde la subjetividad es el plano de fondo, y con ello el interés de reivindicar las luchas de los pueblos indígenas en Colombia para que se valoren y legitimen otras formas de educación no disciplinar. Estas las podríamos llamar de educación indisciplinar, que viene desde las propias prácticas educativas de los pueblos y que considera las particularidades e intereses de cada una de las culturas.

Aquí se transgreden líneas de formación de profesores indígenas, que enseñan matemáticas desterritorializando a la Matemática eurocéntrica en la escuela y territorializándola en otros términos como parte de la decolonialidad del saber y de las apuestas por otras educaciones. Lo anterior hace parte del conjunto de demandas políticas y reclamaciones de autonomía que, desde inicios del siglo XX hasta el presente, vienen planteando al Estado colombiano los grupos étnicos y sus organizaciones. Es importante resaltar estas propuestas innovadoras en un contexto universitario donde todo se presenta de forma disciplinar, pero también es necesario que se hagan más comunes, es decir, que partan del diálogo con las comunidades y que se acoplen a los tiempos y rituales de estas, como Higuita Ramirez (2014) ya señaló.

La Licenciatura en Pedagogía de la Madre Tierra y sus postulados extraídos en los cursos de Etnomatemáticas nos han llevado a conocer otras formas de ser y pensar que pueden dialogar de forma unilateral con los conocimientos matemáticos occidentales, donde la interculturalidad no es un problema, sino que al contrario, es uno de los caminos más eficaces para que nuestras sociedades logren sanar las brechas que nos han separado a unos de otros por la imposición de un modelo único - de cultura, de Universidad, de escuela, de matemática... - basado en la 
diferencia - cultural, de creencias, de prácticas, de raza, de género... - y no en la diversidad.

Por conclusión, es importante promover la construcción de una base latinoamericana de políticas tanto en materia de EIB, específicamente, como de políticas vinculadas a la interculturalización de los sistemas educativos. La información disponible resulta fundamentalmente descriptiva. En algún sentido, las experiencias sobre las que más se ha profundizado resultan repetitivas y poco significativas en términos sustantivos. En Latinoamérica existe un enorme déficit a nivel regional de información circulante sobre las características de las políticas, sus alcances y el porcentaje de financiamiento respecto al presupuesto total del sector educativo, así como los efectos logrados tanto en materia de EIB, específicamente, como en la transversalización intercultural de los sistemas. En general, los datos dispersos e incompletos obstaculizan el análisis de información específica y en profundidad que permita evaluar el panorama, tanto en materia de la EIB, como de los avances dirigidos a los interculturalización de los sistemas educativos.

El desarrollo de la educación intercultural en América Latina se ha constituido en las últimas décadas en un proceso complejo, plural y original. La problemática, propia de un ámbito muy específico, que es la educación escolar indígena, se fue extendiendo hasta encontrarse, hoy, íntimamente ligada a los diferentes proyectos de Estado y de sociedad que están en disputa en los diversos países del continente. Consideramos que el desafío fundamental está en vincular las propuestas de educación intercultural a la perspectiva de la interculturalidad crítica y la etnomatematica.

Es decir, se trata de una tarea compleja, ya que en la mayoría de los países en que se introdujo la interculturalidad en las políticas públicas, sobre todo en el ámbito educacional, por lo general predomina el enfoque funcional y el abordaje aditivo. Estos en muchos casos son folclorizantes y se limitan a incorporar en el currículo escolar componentes de las dos culturas de grupos sociales considerados "diferentes", particularmente indígenas y afrodescendientes.

Pero para que este tema pueda ser trabajado, es fundamental que integre el debate público en diferentes ámbitos sociales. En el caso de la educación, esta discusión aún está poco presente en las instituciones responsables por la formación de educadores, lo que constituye un gran obstáculo para su desarrollo. Sin embargo, las diferencias en las prácticas escolares y en las prácticas de la educación no formal están cobrando una sensibilidad y una visibilidad crecientes, a partir, muchas veces, de situaciones conflictivas. Es decir, el debate sobre la educación intercultural está llamado a afianzarse en las sociedades latinoamericanas, incluso mediante la polémica y el enfrentamiento de posturas.

Finalmente, en primer lugar, debería estar siempre presente este ejercicio ciudadano para 
preparar a los jóvenes y a los futuros profesores para desenvolverse en esferas de cosmovisiones diversas y defendiendo activamente el valor de la igualdad, para desarrollar una actitud de respeto por la diferencia y poder desarrollar la capacidad de entendimiento y del diálogo intercultural latinoamericano.

\section{Referencias citadas:}

Abdallah-Pretceille, M. (1992): Vers une pedagogie interculturelle, Paris, CNDP/Hachette.

Abdallah-Pretceille, M. y L. Porcher (1999): Diagonales de la comunicación interculturelle, Paris, Anthropos.

CEPAL (Comisión Económica para América Latina y el Caribe) (2017): Brechas, ejes y desafíos en el vínculo entre lo social y lo productivo, Santiago (LC/ CDS.2/3).

Corbetta, S. (2016): “Acá tiene la escuela: arréglese”. La migración Qom (toba) y las políticas de Educación Intercultural Bilingüe en la ciudad de Rosario, Santa Fe. Argentina (1990-2014), Tesis de doctorado, Facultad de Ciencias Sociales, Universidad de Buenos Aires, Buenos Aires.

D’Ambrosio, U. (2001): Etnomatemática: Elo entre las tradições e a modernidad, Belo Horizonte, Autêntica.

Demorgen, J. y M. Lipiansky (1999): Guide de l'interculturel en formation, Paris, Retz.
Ferrão Candau, V. M. (2013): "Educación intercultural crítica: Construyendo caminos", en C. Walsh, ed., Pedagogías decoloniales. Prácticas insurgentes de resistir, (re)existir y (re)vivir. Tomo I, Quito, Ediciones Abya-Yala, pp. 145-161.

Fernández, C. (2003): La escuela rural. Interculturalidad mapuche, Buenos Aires, Dunken.

Freire, P. (1997): La pedagogía de la autonomía: saberes necesarios para la práctica educativa, Buenos Aires, Siglo XXI.

Fornet-Betancourt, R. (2001): “Filosofía e interculturalidad en América Latina, intento de introducción no filosófica", Itinerarios: revista de estudios lingüisticos, literarios, históricos y antropológicos, (4), pp. 45-58.

Fornet-Betancourt, R. (2004): Crítica intercultural de la filosofía latinoamericana actual, Madrid, Trotta.

Giroux, H. (1990): Los profesores como intelectuales, Barcelona, Paidos.

Gualdieri, B. (2004): El lenguaje de las experiencias, en MECyT, EIB en Argentina. Sistematización de experiencias, Buenos Aires, MECyT, (507-521).

Higuita Ramírez C., H. Blanco Álvarez y M. L. Oliveras (2014): “Una mirada a la etnomatemática y la educación matemática en Colombia: caminos recorridos", Revista Latinoamericana de etnomatemática, 7(2), pp. 245-269 
Jaramillo, D. (2006): “Interacciones en clase de matemática: una mirada desde la etnomatemática”, en Memorias del Foro Educativo Nacional, Año de Competencias Matemáticas, Bogotá, menc, s.p.

Knijnik, G. (2003): “Currículo, etnomatemática e educação popular: um studo em un asentamento do movimiento Sem Terr a", Curriculo sem Fronteiras, 3(1), pp. 96-110.

Lander, E. (2000): La colonialidad del saber: eurocentrismo y ciencias sociales. Perspectivas Latinoamericanas, Buenos Aires, CLACSO.

Lizcano, E. (2002): “Las matemáticas de la tribu europea: un estudio de caso", en II Congresso Internacional de Etnomatemática (inédito), OuroPreto, Red Latinoamericana de Etnomatemática.

López, E. y F. Prada (2004): Educación superior y descentralización epistemológica, Conferencia Internacional sobre Enseñanza Superior Indígena (texto inédito), Brasil, Universidad de Mato Grosso.

López, L. E. (2009): Interculturalidad, educación y ciudadanía. Perspectivas latinoamericanas, La Paz, Plural editores.

Mato, D. (2008): “Diversidad cultural e interculturalidad en educación superior. Experiencias en América Latina”, Caracas, IESALC-UNESCO, 182, 8(23).

Mato, D. (2011): "Universidades indígenas en América Latina: logos, problemas y desafíos", Revista Andaluza de Antropología, 1, pp. 63-85.
Maya, A. (1996): El reto de la vida. Ecosistemas y cultura, una introducción al estudio del medio ambiente, Bogota, ECOFONDO.

Monteiro, A., D. Orey y M. Domite (2004): Etnomatemática: papel, valor e significado, São Paulo, Zouk.

Moya, R. (2009): "La interculturalidad para todos en América Latina”, en E. López, Ed., Interculturalidad,educación y ciudadanía, Perspectivas latinoamericanas, La Paz, Plural Editores, pp. 21-54.

Novaro, G. (2004): "Pueblos indígenas y escuela. Avances y obstáculos para el desarrollo de un enfoque intercultural”, EIB en Argentina. Sistematización de experiencias, ME, Buenos Aires.

OREALC/UNESCO (Organización de las Naciones Unidas para la Educación, la Ciencia y la Cultura) (2017): Inequidad en los logros de aprendizaje entre estudiantes indígenas en América Latina: ¿Qué nos dice TERCE?, Santiago de Chile, Naciones Unidas.

Osorio, J. (2007): “A joint message from the director of Kamakakûokalani Center for Hawaiian Studies and coordinator of Kawaihuelani Hawaiian Language Program", en Hawai'inuiâkea School of Hawaiian Knowledge, University of Hawai' I, Manoa (inédito).

Quilaqueo, D., S. Quintriqueo, D. Catriquir y G. Llanquinao (2002): Kimeltuwün mew amukey ta zugu: una dialéctica para abordar el conocimiento mapuche en el proceso de formación inicial en educación intercultural, Temuco, Universidad Católica de Temuco, Vicerrectoria Académica. 
Quilaqueo, D., S. Quintriqueo y P. Cárdenas (2005): Educación curriculum e interculturalidad: elementos sobre formación de profesores en contexto mapuche, Temuco, Universidad Católica de Temuco, Facultad de Educación.

Rebolledo, N. (2005): “Interculturalismo y autonomía. Las universidades indígenas y las políticas de alteridad”, en C. Navarro, Coord., La mala educación en tiempos de la derecha, México, Universidad Pedagógica Nacional-Miguel Ángel Porrúa.

SITEAL (Sistema de Tendencias Educativas de América Latina) (2011): La educación de los pueblos indígenas y afrodescendientes, Buenos Aires, IIPE-UNESCO.

Tubino, F. (2005). "La interculturalidad crítica como proyecto ético-político", en Encuentro continental de educadores agustinos, Lima.

UNESCO (Organización de las Naciones Unidas para la Educación, la Ciencia y la Cultura) (2015): Replantear la educación ¿Hacia un bien común mundial?, París, UNESCO.

Walsh, C. y L. Edizon (2007): "Afro Andean Thought and Diasporic Ancestrality”, en M. Banchetti y C. Headley (edits.), Shifting the Geography of Reason: Gender; Science, and Religion, Londres, Cambridge Scholars, pp. 211-224.

Walsh, C. (2009), Interculturalidad, Estado, sociedad. Luchas (de) coloniales de nuestra época, Quito, Ediciones Abya Ayala. 